\title{
LES VIOLATIONS DU DROIT INTERNATIONAL HUMANITAIRE DANS LE CONFLIT DE L'ÉTÉ 2006 AU LIBAN
}

\author{
Rafael Bustos \\ Ministère espagnol de l'Éducation \\ Chercheur invité à l'IREMAM
}

Le droit international, comme toute forme de droit mais plus encore parce qu'il est un droit interétatique, est une construction du pouvoir ou des pouvoirs. En même temps qu'il est à chaque moment l'expression d'un certain rapport de forces, il crée des normes, des minimums moraux que les moins puissants peuvent invoquer à leur bénéfice. À l'époque actuelle, le droit international public (DIP) qui a pris des décennies pour se former est en train d'être démantelé à une vitesse surprenante. Il est soit miné par des transgressions (des "guerres illégales" ou des interventions armées sans autorisation, par exemple), soit appliqué de manière suis generis quand son application n'est pas simplement contraire au droit (comme dans les guerres et invasions préventives ou le prétendu nouveau statut des prisonniers de Guantánamo).

Dans ce contexte d'érosion des critères d'application de la législation internationale, le DIP et le droit international humanitaire $(\mathrm{DIH})$ deviennent précieux tant comme instruments à la portée des adversaires que comme cadre régulateur des hostilités. Les sociétés, surtout celles qui ont eu à en souffrir, manifestent un désir profond de fixer et faire valoir les principes consolidés du $\mathrm{DIH}$, d'instaurer une espèce de justice après l'injustice qu'est la guerre. Mais cette aspiration légitime a quelque chose d'irréel parce que le DIH continue à relever des mécanismes communs au DIP, et donc, du pouvoir.

\section{Le droit international humanitaire et son application au conflit israélo-libanais}

Le droit international humanitaire est composé d'un corps de traités et de coutumes internationales qui ont été progressivement codifiés à partir du XIXe siècle. Il englobe le droit de la guerre (dit «droit de La Haye») et le droit humanitaire stricto sensu (dit «droit de Genève »), fusionnés par le premier Protocole additif aux Conventions de Genève en 1977. La 
création de tribunaux pénaux internationaux, spéciaux ou ad hoc, et généraux ou permanents (comme la Cour Pénale Internationale, CPI) depuis la deuxième guerre mondiale a ajouté des nouvelles normes qui complètent et renforcent le DIH.

À la différence du DIP qui engage la responsabilité des États à qui appartient le privilège de le mettre en oeuvre, dans le DIH existe en outre la responsabilité pénale internationale, qui permet l'incrimination et la sanction des individus auteurs de violations sur la demande d'autres individus ou d'un État. Les violations d'extrême gravité susceptibles de répression pénale sont le génocide, le crime contre l'humanité, les crimes de guerre - définis comme des "infractions graves" au DIH ${ }^{1}$ - et peut-être, dans le futur, le crime d'agression.

Quel est le droit international humanitaire applicable au conflit israélo-libanais? Il existe deux possibilités. On applique soit l'article 3 commun aux Conventions de Genève, qui couvre tous les conflits entre un État et un acteur non-étatique ; soit, en se basant sur les déclarations d'Israël sur le conflit (par exemple, sa déclaration de guerre au Liban), on applique les quatre Conventions de Genève et surtout le premier Protocole additif relatif aux conflits internationaux. Peu importe qu'Israël n'ait pas ratifié ce Protocole car la majorité écrasante de ses dispositions sont devenues coutume internationale et sont par conséquent opposables à tous les États. Dans un cas comme dans l'autre, il n'y a pas grande différence concernant la norme à tenir dans la conduite des hostilités ni dans l'obligation de la respecter.

\section{Les violations du droit international humanitaire pendant le conflit}

À défaut d'une enquête internationale qui détermine les faits durant le conflit israélo-libanais, nous distinguerons les violations plus documentées et plus probables (crimes de guerre présumés) des violations moins documentées ou plus discutables (crimes de guerre éventuels).

-Crimes de guerres présumés

"L’attaque délibérée" contre des objectifs et des populations civiles est un crime de guerre. Or, l'évidence paraît indiquer qu'aussi bien Israël que le Hezbollah l'ont pratiquée. Dans le cas

\footnotetext{
${ }^{1}$ Les personnes protégées sont les combattants blessés ou malades ou naufragés, les prisonniers de guerre et les civils.
} 
d'Israël, des attaques contre de convois de civils qui fuyaient, contre un camp de réfugiés et contre plusieurs localités ont été constatées. Le bombardement d'habitations civiles comme par exemple celles de Cana, Aïtaroun ou Tyr appartiennent bien sûr à cette catégorie.

Des biens publics ont été aussi des cibles de l'armée israélienne, tels qu'une centrale électrique, des canalisations d'eau, des hôpitaux et des usines alimentaires. Nombre d'attaques bien documentées n'ont pas eu d'objectif militaire. En retour, le Hezbollah a lancé un quart de ces rockets et missiles contre des localités, dans beaucoup desquelles il n'existait pas d'objectif militaire. Par conséquent des écoles, des hôpitaux et des logements ont été atteintes dans différents points du nord d'Israël (voir par exemple le rapport d'Amnéstie Internationale, http://web.amnesty.org/library/Index/ENGMDE020252006). Ces faits constituent des crimes de guerre. En aucun de ces cas l'erreur ou l'accident ne pourrait être invoqué comme excuse ou circonstance atténuante, puisque la partie attaquante est autant responsable de la manœuvre du tir que de ses conséquences. ${ }^{2}$

"L'attaque intentionnelle contre le personnel ou des installations d'aide humanitaire de même que contre le personnel associé aux missions de paix” fait partie des crimes de guerre. En dépit de cela, on a registré des opérations israéliennes contre les installations et le personnel de la FINUL, le personnel de la Croix Rouge et ses véhicules, ainsi que contre le personnel de l'UNRWA, entraînant la mort de plusieurs personnes. Au moins en une occasion, une rocket du Hezbollah a atteint des installations de la FINUL, sans causer de victimes.

"L'attaque indiscriminée" est celle qui ne permet pas de discerner des objectifs militaires de la population et des objectifs civils, en raison soit de la proximité, soit de la densité de population. Tandis que "l'attaque disproportionnée" est celle où l'intensité des tirs de projectiles ou la dispersion des lancements rendent impossible d'éviter les dommages aux civils. Les deux parties auraient commis ces types de crimes de guerre. ${ }^{3}$ Le bombardement israélien sur les quartiers du sud de Beyrouth (notamment, Haret Hreik), dont l'objectif déclaré était le siège du Hezbollah et

\footnotetext{
${ }^{2}$ Voir par exemple le précédent de l'Arrangement du 26 avril 1996 et les rapports du Groupe de Surveillance. E. Canal-Forgues, « La surveillance de l'application de l'arrangement du 26 avril 1996 (Israël-Liban)», Revue Générale de Droit International Public, juillet-septembre 1998, n³, p.744.

${ }^{3}$ Le nombre d'attaques indiscriminées et/ou disproportionnées n'est pas connu encore, mais en revanche on peut se faire une idée de l'ampleur globale des offensives et du nombre de victimes civiles. Une moyenne de 3000 bombes ont été tirées sur le Liban par jour, tandis que le Hezbollah a tiré environ 4000 rockets et petit missiles pendant tout le conflit sur Israël, dont une partie a été interceptée. On sait aussi que cette guerre a fait d'une part 1183 morts civils et entre 91 et 134 combattants de nationalité libanaise (toutes forces armées confondues) et d'autre part, 52 civils et 118 soldats de nationalité israélienne. Source: Nations Unies (Rapport S/2006/670 de 18 août 2006 et Daily Star online, www.dailystar.com.lb 29 août 2006.
} 
la chaîne de télévision al-Manar a été une attaque indiscriminée et disproportionnée par son intensité ( 23 tonnes de bombes en un seul jour, au moins 127 bâtiments détruits) et par la densité de la population qui entourait les cibles. Quant au Hezbollah, la plupart des tirs contre les communes du nord d'Israël ont été réalisés avec des armes qui ne permettaient pas de distinguer entre objectifs militaires et civils. La distance, l'absence de système de guidage et la masse en métal parfois rajoutée rendaient impossible toute précision. Ces tirs exposaient donc des civils et des militaires au même risque, et furent donc indiscriminés.

Israël a justifié ses opérations contre des objectifs mixtes en opposant l'avantage militaire "global" aux dégâts humains. Le calcul de cet avantage n'est pas basé sur une opération concrète mais sur tout le déroulement du conflit, c'est à dire, sur l'usage éventuel que le Hezbollah pourrait faire un jour de toutes ses armes s'il déclenchait une seule attaque contre Israël. Cette interprétation très particulière de l'avantage militaire face au coût civil est indéfendable au regard du DIH. L'Institut de Droit International a fixé depuis longtemps la doctrine selon laquelle l'avantage militaire devait être "concret et immédiat", pas abstrait ni futur (Rés. 9 de Sept. de 1969).

L'utilisation de "certaines armes qui peuvent causer un dommage massif à la population civile" est bannie. Malgré cela, Israël a employé des bombes à sous-munitions, fournies par les États Unis, à grande échelle. Ces bombes sont caractérisées par une grande dispersion et un pourcentage élevé d'erreur. Le DIH interdit leur usage "dans des endroits habités ou près des populations". Les premières victimes de ces engins sont apparues dès la fin de la guerre. Le minage indiscriminé, tel qu'il a pu être réalisé par Israël rentre aussi dans cette catégorie de prohibitions. Dans le cas du Hezbollah, l'utilisation de masses de métal dans les rockets pour redoubler l'impact viole la prohibition générale de ne pas causer de "maux superflus".

-Crimes de guerres éventuels

“Lancer des attaques en sachant qu'elles peuvent occasionner des préjudices écologiques graves, étendus et durables" est interdit par le DIH. Or, Israël a détruit un dépôt de pétrole à la centrale électrique de Jiyeh sur la côte libanaise, à usage civil. Ce dépôt contenait 35.000 tonnes de fuel, dont une partie a brûlé et une autre s'est déversée en mer causant une pollution de grande ampleur. 
"Stocker de l'armement dans des endroits habités et lancer des attaques à partir d'eux ou près des observateurs internationaux" est interdit, ainsi que "diriger la population ou la retenir pour protéger des installations militaires du feu ennemi" (boucliers humains). Alors qu'il n'existe pas d'évidence directe de l'usage de "boucliers humains" par le Hezbollah, il y a des indices (tel que le rapport de HRW, sur http://hrw.org/reports/2006/lebanon0806/lebanon0806web.pdf, p.3) selon lesquels la milice chiite aurait gardé des armes près des maisons tandis que ses combattants auraient placé occasionnellement des lance rockets à l'intérieur des localités ou à proximité des postes d'observation des Nations Unies. Ce fait-ci ne justisfie absolument pas les attaques indiscriminées contre les localités concernées.

“Faire obstacle ou empêcher délibérément l'accès des victimes de guerre aux secours" est aussi un crime de guerre. Israël a empêché à plusieurs reprises le passage des convois et véhicules d'aide humanitaire. L'annonce faite par l'armée israélienne en vertu de laquelle "tout véhicule circulant au sud de la rivière Litani" serait bombardé par l'aviation a mis en grave danger les organisations internationales de secours. Le maintien du blocus maritime et aérien quelques semaines après le cessez-le-feu a également rendu impossible l'accès à de nombreuses localités privées de communication par la destruction de ponts et routes.

"Endommager de façon générale et délibérée des propriétés quand cette opération n'est pas justifiée par des buts militaires", est aussi un crime de guerre. Un recensement de l'étendue, de la localisation et du nombre des propriétés ravagées déjà en cours est nécessaire pour constater la transgression par l'État israélien de cette norme internationale, de même qu'une analyse des objectifs militaires des opérations aériennes et terrestres qui ont provoqué leur destruction.

\section{La répression des violations du DIP et des crimes de guerre}

En ce qui concerne le droit international public, la répression relève de la responsabilité internationale des États, selon le principe de "réparation des dommages occasionnés". Afin que cette responsabilité soit admise, il faut qu'un État dépose une plainte contre un autre. Dans cette guerre, le Liban peut mettre en cause la responsabilité internationale d'Israël pour les dégâts moraux et matériels aux infrastructures, industries, services, zones littorales, propriétés et d'autres intérêts économiques (tel que le tourisme ou la pêche). Des précédents existent dans ce 
sens, la Cour Internationale de Justice (CIJ) ayant imposé en 2005 à l'Ouganda la réparation de dommages dérivés de son occupation illégale du Congo, y compris le pillage de ressources naturelles. Mais aussi le cas des indemnités versées par le gouvernement et des sociétés allemandes aux citoyens juifs, ou celui de l'Irak aux victimes de l'invasion de Koweït de 1991. Dans la pratique, néanmoins, il est difficile que cette responsabilité soit reconnue par l'État en infraction, à moins qu'il accepte de se soumettre à une juridiction internationale (la CIJ, par exemple) ou qu'elle soit retenue par le Conseil de Sécurité (cas de l'Irak).

Pour la répression des crimes de guerre, trois voies de réclamation se dessinent. La première opère à l'échelle nationale et repose sur l'obligation des États d'adopter dans leur système juridique interne les instruments pénaux qui permettent l'application du DIH. Or, il n'est pas courant qu'un État en infraction enquête et juge un de ses citoyens pour crimes de guerre. Il est aussi possible, une fois épuisée la démarche précédente, que sous accord de deux États, une commission libano-israélienne soit créée, comme cela s'est fait ailleurs. Cette possibilité passe par d'éventuelles réclamations des familles dont les membres ou propriétés ont été attaquées sans cause apparente (existence dans les environs d'installations militaires ou de combats).

La deuxième voie est basée sur le principe aut judicare aut dedere (faire appliquer le droit), selon lequel tous les États doivent "respecter et faire respecter" les normes de DIH, quels que soient les auteurs où le lieu de l'infraction. En plus, pour un tiers à peu près des États du monde (51), les crimes de guerre et le génocide sont des crimes imprescriptibles ${ }^{4}$ et peuvent être poursuivis à n'importe quel moment. Une juridiction interétatique pour ce type de crimes serait par conséquent en train de s'ériger. Au cas où ni Israël ni le Liban ne veulent ou ne peuvent juger ces crimes, il n'est pas exclu que le tribunal pénal d'un autre pays accepte un jour de le faire.

Finalement, la troisième voie est celle des organisations internationales, particulièrement, la CPI, dont le statut prévoit que le Conseil de Sécurité puisse lui demander d'entamer une enquête sur une possible violation de droit international concernant, par sa gravité, l'humanitaire. Cela est possible même si l'État en infraction n'est pas partie au statut de la CPI (ni Israël ni le Liban ne le sont). Par ailleurs, le Secrétaire Général de Nations Unies est aussi autorisé à diligenter une enquête internationale sur des faits ayant entraîné des violations graves du DIH. Si l'on

\footnotetext{
${ }^{4}$ En vertu de deux traités, la Convention de Nations Unies de 1968 et la Convention européenne de 1974 sur l'imprescriptibilité des crimes de guerre et du génocide.
} 
considère le nombre de données documentées par les organisations internationales (Comité International de la Croix Rouge, Human Rights Watch, Amnesty International, etc.), une telle enquête internationale serait le point de départ le plus approprié.

\section{Raports cités :}

Amnesty International. Israel/Lebanon Under fire: Hizbullah's attacks on northern Israel, MDE 02/025/2006, 14 September 2006, sur http://web.amnesty.org/library/Index/ENGMDE020252006.

Human Rights Watch, Fatal Strikes. Israel's Indiscriminate Attacks Against Civilians in Lebanon. August 2006, volume 18, No.3 (E), sur http://hrw.org/reports/2006/lebanon0806/lebanon0806web.pdf. 